\title{
Two-Dimensional Nonlinear Reaction Diffusion Equation with Time Efficient Scheme
}

\author{
Shahid Hasnain, Muhammad Saqib, Daoud Suleiman Mashat \\ Department of Mathematics, Numerical Analysis, King Abdulaziz University, Jeddah, KSA \\ Email:dmashat@kau.edu.sa, shahidqa32@gmail.com
}

How to cite this paper: Hasnain, S., Saqib, M. and Mashat, D.S. (2017) Two-Dimensional Nonlinear Reaction Diffusion Equation with Time Efficient Scheme. American Journal of Computational Mathematics, 7, 183-194.

https://doi.org/10.4236/ajcm.2017.72017

Received: January 31, 2017

Accepted: June 20, 2017

Published: June 23, 2017

Copyright $\odot 2017$ by authors and Scientific Research Publishing Inc. This work is licensed under the Creative Commons Attribution International License (CC BY 4.0).

http://creativecommons.org/licenses/by/4.0/

\begin{abstract}
This research paper represents a numerical approximation to non-linear two-dimensional reaction diffusion equation from population genetics. Since various initial and boundary value problems exist in two-dimensional reaction-diffusion, phenomena are studied numerically by different numerical methods, here we use finite difference schemes to approximate the solution. Accuracy is studied in term of $L_{2}, L_{\infty}$ and relative error norms by random selected grids along time levels for comparison with exact results. The test example demonstrates the accuracy, efficiency and versatility of the proposed schemes. It is shown that the numerical schemes give better solutions. Moreover, the schemes can be easily applied to a wide class of higher dimension nonlinear reaction diffusion equations with a little modification.
\end{abstract}

\section{Keywords}

Forward in Time and Centre in Space (FTCS), Taylor's Series, Crank Nicolson, Alternating Direction Implicit (ADI) Scheme

\section{Introduction}

Let us consider a population distributed in a linear habitat, such as shore line, which occupies with a uniform quantity of density [1]. If at any point of the habitat, a mutation occurs, which happens to be in some degree, however, slight, advantageous to survival, in the totality of its effects [1] [2]. We may expect the mutant gene to increase at the expense of all allelomorph or allelomorphs previously occupying the same set of points [2] [3]. This process will be first computed in the neighbourhood of the occurrence of the mutation and later, as the advantageous gene is diffused into the surrounding population, in the adjacent portions of its range [3]. Supposing that this range to be long compared with the distances separately, the sites of offspring from these of their parents, 
there will be advancing from origin, a wave of increase in the gene frequency [4]. Let us consider the following possible postulates of above phenomena.

Let $p$ be the frequency of the mutant gene, and $q$ is its parent allelomorph and we suppose, it is only the allelomorph parent [4] [5]. Let $m$ be the intensity of the selection in favour of the mutant gene, supposing independence of $p$. Let us suppose that the rate of diffusion per generation across any boundary may be equated to $-k \partial_{x} p$ at that boundary, $x$ being the coordinate measuring position in the linear habitat [5]. Then $p$ must satisfy the differential equation,

$$
p_{t}=k p_{x x}+m p q
$$

where $t$ represents time in generation, where constant $k$ is coefficient of diffusion analogous which is used in physics.

\section{Governing Equation}

Many complicated natural phenomena, such as the spreading of bushfire and epidemics, and the non-linear evolution of a population in a two dimensional habitat [6] [7] [8] [9], (in which the balance of reaction and diffusion are concerned) can be modelled by a two dimensional reaction-diffusion equation

$$
\left.\begin{array}{c}
u_{t}=\boldsymbol{\beta}\left(u_{x x}+u_{y y}\right)+\alpha f(u) \\
u_{t}=\nabla \cdot(\boldsymbol{\beta} \nabla u)+\alpha f(u)
\end{array}\right\}
$$

where $u$ is a dimensionless temperature or population density, $u_{t}$ is the rate of increase of $u$ with time $t, \nabla$ is the gradient operator in two dimensional space, $\beta$ is a constant second order tensor measuring the diffusivity of the media, and $f(u)$ is a non-linear function of $u$ representing the effect of reaction or multiplication. Also $\mu$ represents reactive constant after diffusion occurs. Assuming $\beta_{1}$ and $\beta_{2}$ are the principal values of $\beta$ as in Equation (2) and $x$ and $y$ are coordinates along the principle axes, Equation (2) can be written as

$$
u_{t}=\beta_{1} u_{x x}+\beta_{2} u_{y y}+\alpha u(1-u)
$$

\section{Exact Solution}

To derive the exact solution of the given system in Equation (3), we assume the exact solution of the two dimensional non-linear reaction diffusion equation is [10] [11] [12] [13],

$$
\left.\begin{array}{r}
\left.u(x, y, t)=20+80\left(y-e^{D t} \sin \left(\frac{x \pi}{2}\right) \sin \left(\frac{y \pi}{2}\right)\right)\right) \\
\text { Where } D=-\frac{\beta \pi^{2}}{2}
\end{array}\right\}
$$

\section{Numerical Methods}

We consider the numerical solution of the non-linear Equation (3) in a finite 
domain $\Omega=\{(x, y) \mid a<x<b, c<y<d\}$. The first step is to choose integers $\mathrm{n}$ and $m$ to define step sizes $h=(b-a) / n$ and $k=(d-c) / m$ in $x$ and $y$ directions respectively. Partition the interval $[a, b]$ into $n$ equal parts of width $h$ and the interval $[c, d]$ into $m$ equal parts of width $k$. Place a grid on the rectangle $R$ by drawing vertical and horizontal lines through the points with coordinates $\left(x_{i}, y_{j}\right)$, where $x_{i}=a+i h$ for each $i=0,1,2, \cdots n$ and $y_{j}=c+j k$ for each $j=0,1,2, \cdots m$ also the lines $x=x_{i}$ and $y=y_{j}$ are grid lines, and their intersections are the mesh points of the grid. For each mesh point in the interior of the grid, $\left(x_{i}, y_{j}\right)$, for $i=1,2, \cdots, n-1$ and $j=1,2, \cdots, m-1$, we apply different algorithms to approximate the numerical solution to the problem in Equation (3) also we assume $t_{N}=N t, N=0,1, \cdots$, where $t$ is the time.

\subsection{Second Order Implicit Scheme}

We apply Crank Nicolson implicit finite difference scheme to Equation (3),

$$
\left.\begin{array}{c}
\delta_{x}^{2} \hat{u}=\frac{\hat{u}_{l+1, m}-2 \hat{u}_{l, m}+\hat{u}_{l-1, m}}{h^{2}} \\
\delta_{y}^{2} \hat{u}=\frac{\hat{u}_{l, m+1}-2 \hat{u}_{l, m}+\hat{u}_{l, m-1}}{h^{2}} \\
\delta_{x}^{2} \hat{v}=\frac{\hat{v}_{l+1, m}-2 \hat{v}_{l, m}+\hat{v}_{l-1, m}}{h^{2}} \\
\delta_{y}^{2} \hat{v}=\frac{\hat{v}_{l, m+1}-2 \hat{v}_{l, m}+\hat{v}_{l, m-1}}{h^{2}}
\end{array}\right\}
$$

\subsection{Computationally Efficient Implicit Scheme}

In search of a time efficient alternate, we analysed the naive version of the Crank-Nicolson scheme for the two dimensional equation, and find out that scheme is not time efficient [14] [15] [16] [17] [18]. To get high time efficiency, the common name of Alternating Direction Implicit (ADI) method, can be used. The derivation to ADI scheme, we have following steps;

\section{ADI formulation: Peaceman-Rachford algorithm:}

Introduce an FTCS scheme for the first time step $\star=n+\frac{1}{2}$, of the form 


$$
\begin{aligned}
& \frac{u_{i, j}^{\star}-u_{i, j}^{n}}{\Delta t / 2}-\frac{\beta_{1}}{h_{x}^{2}}\left(u_{i-1, j}^{\star}-2 u_{i, j}^{\star}+u_{i+1, j}^{\star}\right) \\
& -\frac{\beta_{2}}{h_{y}^{2}}\left(u_{i, j-1}^{n}-2 u_{i, j}^{n}+u_{i, j+1}^{n}\right)=\mu f\left(u_{i, j}^{n}\right)
\end{aligned}
$$

in the compact form, above equation can be seen as:

$$
\frac{u_{i, j}^{\star}-u_{i, j}^{n}}{\Delta t / 2}-S_{1} L_{x x} u_{i, j}^{\star}-S_{2} L_{y y} u_{i, j}^{n}=\mu f\left(u_{i, j}^{n}\right)
$$

above equation can be written as in algorithm form, for $i=1,2,3, \cdots, n-1$

$$
\begin{aligned}
& -\frac{1}{2} \boldsymbol{R}_{1} u_{i-1, j}^{\star}+\left(1+\boldsymbol{R}_{1}\right) u_{i, j}^{\star}-\frac{1}{2} \boldsymbol{R}_{1} u_{i+1, j}^{\star} \\
& =\frac{1}{2} \boldsymbol{R}_{2} u_{i, j-1}^{n}+\left(1-\boldsymbol{R}_{2}\right) u_{i, j}^{n}+\frac{1}{2} \boldsymbol{R}_{2} u_{i, j+1}^{n}+\frac{\mu \Delta t}{2} f\left(u_{i, j}^{n}\right)
\end{aligned}
$$

above algorithm validates its results for $i=1,2,3, \cdots, n-1$ )

Now let us consider the second time step,

$$
\begin{aligned}
& \frac{u_{i, j}^{n+1}-u_{i, j}^{\star}}{\Delta t / 2}-\frac{\beta_{1}}{h_{x}^{2}}\left(u_{i-1, j}^{\star}-2 u_{i, j}^{\star}+u_{i+1, j}^{\star}\right) \\
& -\frac{\beta_{2}}{h_{y}^{2}}\left(u_{i, j-1}^{n+1}-2 u_{i, j}^{n+1}+u_{i, j+1}^{n+1}\right)=\mu f\left(u_{i, j}^{\star}\right)
\end{aligned}
$$

in the compact form, above equation can be seen as:

$$
\begin{aligned}
& \left.\begin{array}{l}
\frac{u_{i, j}^{n+1}-u_{i, j}^{\star}}{\Delta t / 2}-S_{1} L_{x x} u_{i, j}^{\star}-S_{2} L_{y y} u_{i, j}^{n+1}=\mu f\left(u_{i, j}^{\star}\right) \\
\quad \text { above equation can be written as in } \\
\quad \text { algorithm form, for } j=1,2,3, \cdots, m-1
\end{array}\right) \\
& -\frac{1}{2} \boldsymbol{R}_{2} u_{i-1, j}^{n+1}+\left(1+\boldsymbol{R}_{2}\right) u_{i, j}^{n+1}-\frac{1}{2} \boldsymbol{R}_{2} u_{i+1, j}^{n+1} \\
& =\frac{1}{2} \boldsymbol{R}_{1} u_{i-1, j}^{\star}+\left(1-\boldsymbol{R}_{1}\right) u_{i, j}^{\star}+\frac{1}{2} \boldsymbol{R}_{1} u_{i+1, j}^{\star}+\frac{\mu \Delta t}{2} f\left(u_{i, j}^{\star}\right)
\end{aligned}
$$

above algorithm validates its results for $j=1,2,3, \cdots, m-1$ )

The trick used in constructing the ADI scheme is to split time step into two, and apply two different stencils in each half time step, therefore to increment time by one time step in grid point, we first compute both of these stencils are chosen such that the resulting linear system is block tridiagonal [19] [20] [21] [22]. To obtain the numerical solution, we need to solve a block non-linear tridiagonal system at each time step. We have done this by using Newton's iterative method.

\section{Algorithm 1:}

The non-linear system in Equations (6) and (7), can be written in the form:

$$
R(W)=0
$$

where $R=\left(r_{1}, r_{2}, r_{3}, \cdots, r_{2 n}\right)^{t}, W=\left(u_{1}^{n+1}, v_{1}^{n+1}, u_{2}^{n+1}, v_{2}^{n+1}, \cdots, u_{m}^{n+1}, v_{m}^{n+1}\right)$ and $r_{1}, r_{2}, r_{3}, \cdots, r_{2 n}$ are the non-linear equations obtained from the system (6 and 7). The system of equations, is solved by Newton's iterative method using the 
following steps

1) Specify $W^{(0)}$ as an initial approximation.

2) For $k=0,1,2, \cdots$ until convergence achieve.

- Solve the linear system $A\left(W^{(k)}\right) \Delta W^{(k)}=-R\left(W^{(k)}\right)$,

- Specify $W^{(k+1)}=W^{(k)}+\Delta W^{(k)}$,

where $A\left(W^{(k)}\right)$ is $(m \times m)$ Jacobian matrix, which is computed analytically and $\Delta W^{(k)}$ is the correction vector. In the iteration method solution at the previous time step is taken as the initial guess. Iteration at each time step is stopped when $\left\|R\left(W^{(k)}\right)\right\|_{\infty} \leq$ Tol with $T o l$ is a very small prescribed value. The linear system obtained from Newton's iterative method, is solved by Crout's method. Convergence done with iterations along less CPU time [23].

\section{Algorithm 2:}

Clearly, the system is tridiagonal and can be solved with Thomas algorithm. The dimension of $J$ is $n \times m$. In general a tridiagonal system can be written as,

$$
a_{i} x_{i-1}+b_{i} x_{i}+c_{i} x_{i+1}=S_{i} \text {, with } a_{1}=c_{n}=0
$$

above system can be written as in a matrix-vector form,

$$
J u=S
$$

where $J$ is a coefficient matrix (Jacobean Matrix), which is known, comes from Newton's iterative method. Right hand side is column vector which is known. Our main goal is to find the resultant vector $u$. Now we have

$$
\begin{aligned}
J= & =\left[\begin{array}{cccccc}
b_{1} & c_{1} & 0 & 0 & 0 & \cdots 0 \\
a_{2} & b_{2} & c_{2} & 0 & 0 & \cdots 0 \\
\cdots & \cdots & \cdots & \cdots & \cdots & \cdots \\
\cdots & \cdots & \cdots & \cdots & \cdots & \cdots \\
\cdots & \cdots & \cdots & \cdots & \cdots & \cdots \\
\cdots & \cdots & \cdots & a_{n-1} & b_{n-1} & c_{n-1} \\
\cdots & \cdots & \cdots & \cdots & a_{n} & b_{n}
\end{array}\right] \\
\underline{u} & =\left[u_{1}, u_{2}, u_{3}, \cdots, u_{n}\right]^{t} \\
\underline{S} & =\left[s_{1}, s_{2}, s_{3}, \cdots, s_{n}\right]^{t}
\end{aligned}
$$

technique is explained in the following steps,

$$
J=L U
$$

where

$$
L=\left[\begin{array}{cccccc}
\mu_{1} & 0 & 0 & 0 & 0 & \ldots 0 \\
\beta_{2} & \mu_{2} & 0 & 0 & 0 & \ldots 0 \\
\alpha_{3} & \beta_{3} & \mu_{3} & 0 & 0 & \ldots 0 \\
\ldots & \ldots & \ldots & \ldots & \ldots & \ldots 0 \\
\ldots & \ldots & \alpha_{n-1} & \beta_{n-1} & \mu_{n-1} & 0 \\
\ldots & \ldots & \ldots & \alpha_{n} & \beta_{n} & \mu_{n}
\end{array}\right]
$$

And 


$$
U=\left[\begin{array}{cccccc}
1 & \delta_{1} & \lambda_{1} & 0 & 0 & \ldots 0 \\
0 & 1 & \delta_{2} & \lambda_{2} & 0 & \ldots 0 \\
0 & 0 & 1 & \delta_{3} & \lambda_{3} & \ldots 0 \\
\cdots & \ldots & \ldots & \cdots & \ldots & \ldots 0 \\
\cdots & \ldots & 0 & 1 & \delta_{n-2} & \lambda_{2} \\
\cdots & \ldots & 0 & 0 & 1 & \delta_{n-1} \\
\cdots & \ldots & \ldots & 0 & 0 & 1
\end{array}\right]
$$

By equating both sides of the $J u=S$, we get the elements of the matrices $L$ and $U$. The computational tricks for the implementation of Thomas algorithm are shown in results, taken from a specific examples.

\section{Error Norms}

The accuracy and consistency of the schemes is measured in terms of error norms specially $L_{2}$ and $L_{\infty}$ [23] [24] [25] [26] which are defined as:

$$
\left.\begin{array}{r}
L_{\infty}=\left\|u^{\text {ecact }}-u^{\text {Approximation }}\right\|_{\infty}=\max _{1 \leq i \leq m} \sum_{j=1}^{m}\left|u_{j}^{\text {ecact }}-u_{j}^{\text {Approximation }}\right| \\
L_{2}=\left\|u^{\text {ecact }}-u^{\text {Approximation }}\right\|_{2}=\sqrt{\sum_{j=1}^{m}\left|u_{j}^{\text {ecact }}-u_{j}^{\text {Approximation }}\right|} \\
\text { Error }_{\text {relative }}=\sqrt{\frac{\sum_{i} \sum_{j}\left|u_{i, j}^{\text {ecact }}-u_{i, j}^{\text {Approximation }}\right|^{2}}{\sum_{j}\left|u_{i, j}^{\text {exact }}\right|^{2}}} \\
\text { Rate }=\frac{\log \left(\text { Error }_{h} / \text { Error }_{h / 2}\right)}{\log (h /(h / 2))}
\end{array}\right\}
$$

\section{Results}

Numerical computations have been performed using the uniform grid. Table 1 \& Table 2 represent results at different grids and time level using Crank Nicolson implicit scheme. We fixed some parameters such as time step $k=0.0001, \quad \beta_{1}=\beta_{2}=1$ and $\beta_{1}=\beta_{2}=1 / 4$. Scheme convergence viewed through $L_{2}, L_{\infty}$, relative error norms [27] [28] [29] [30]. Table 3 \& Table 4 represent results at different grids and time level using ADI implicit scheme, keeping fixed parameters as we did before. In Table 5, we get results using ADI

Table 1. Estimates of results using Crank Nicolson with some fixed parameters such as $k=0.0001, t=1$ at different grids and $[a, b]=\left[\begin{array}{ll}-10 & 10\end{array}\right]$. Error magnifies through $L_{2}$, $L_{\infty}$ and Error $_{\text {relative }}$.

\begin{tabular}{cccccccc}
\hline & \multicolumn{3}{c}{$\beta_{1}=\beta_{2}=1$} & \multicolumn{3}{c}{$\beta_{1}=\beta_{2}=1 / 4$} \\
\hline \multirow{2}{*}{ Time $=t$} & $x$ & $L_{2}$ & $L_{\infty}$ & $L_{\text {relative }}$ & $L_{2}$ & $L_{\infty}$ & $L_{\text {relaive }}$ \\
\hline \multirow{2}{*}{1} & $21 \times 21$ & 0.0639 & 0.0109 & 0.0099 & 0.0531 & 0.0109 & 0.0097 \\
& $51 \times 51$ & 0.0313 & 0.0059 & 0.0043 & 0.0213 & 0.0051 & 0.0031 \\
& $101 \times 101$ & 0.0112 & 0.0017 & 0.0009 & 0.0115 & 0.0021 & 0.00099 \\
\hline
\end{tabular}


Table 2. Estimates of results using Crank Nicolson with some fixed parameters such as $k=0.0001$, grid $=61 \times 61$ at different time level and $[a, b]=\left[\begin{array}{ll}-10 & 10\end{array}\right]$. Error magnifies through $L_{2}, L_{\infty}$ and Error $_{\text {relative }}$.

\begin{tabular}{cccccccc}
\hline & \multicolumn{3}{c}{$\beta_{1}=\beta_{2}=1$} & \multicolumn{3}{c}{$\beta_{1}=\beta_{2}=1 / 4$} \\
\hline \multirow{2}{*}{ Grid } & $t$ & $L_{2}$ & $L_{\infty}$ & $L_{\text {relative }}$ & $L_{2}$ & $L_{\infty}$ & $L_{\text {relative }}$ \\
\hline \multirow{2}{*}{$61 \times 61$} & 0.2 & 0.0791 & 0.0692 & 0.0543 & 0.0789 & 0.0670 & 0.0512 \\
& 0.4 & 0.0998 & 0.0891 & 0.0753 & 0.0990 & 0.0881 & 0.0615 \\
& 0.6 & 0.1386 & 0.0993 & 0.0991 & 0.1189 & 0.0987 & 0.0899 \\
\hline
\end{tabular}

Table 3. Estimates of results using ADI scheme, such as $k=0.0001, t=1$ at different grids and $[a, b]=\left[\begin{array}{ll}-10 & 10\end{array}\right]$. Error magnifies through $L_{2}, L_{\infty}$ and Error $_{\text {relative }}$.

\begin{tabular}{cccccccc}
\hline & \multicolumn{3}{c}{$\beta_{1}=\beta_{2}=1$} & \multicolumn{3}{c}{$\beta_{1}=\beta_{2}=1 / 4$} \\
\hline Time $=t$ & $X$ & $L_{2}$ & $L_{\infty}$ & $L_{\text {relative }}$ & $L_{2}$ & $L_{\infty}$ & $L_{\text {relative }}$ \\
\hline 1 & $21 \times 21$ & 0.0304 & 0.0211 & 0.0069 & 0.0344 & 0.0209 & 0.0063 \\
& $51 \times 51$ & 0.0110 & 0.0073 & 0.0037 & 0.0210 & 0.0079 & 0.0033 \\
& $101 \times 101$ & 0.0099 & 0.0051 & 0.00099 & 0.0100 & 0.0062 & 0.000891 \\
\hline
\end{tabular}

Table 4. Estimates of results using ADI scheme with some fixed parameters such as $k=0.0001$, grid $=61 \times 61$ at different time level and $[a, b]=\left[\begin{array}{ll}-10 & 10\end{array}\right]$. Error magnifies through $L_{2}, L_{\infty}$ and Error $_{\text {relative }}$.

\begin{tabular}{cccccccc}
\hline & \multicolumn{4}{c}{ Crank Scheme } & \multicolumn{3}{c}{ ADI Scheme } \\
\hline \multirow{2}{*}{ Grid } & $t$ & $L_{2}$ & $L_{\infty}$ & $L_{\text {relative }}$ & $L_{2}$ & $L_{\infty}$ & $L_{\text {relative }}$ \\
\hline \multirow{2}{*}{$61 \times 61$} & 0.2 & 0.0263 & 0.0231 & 0.0181 & 0.0263 & 0.0223 & 0.0171 \\
& 0.4 & 0.0332 & 0.0297 & 0.0251 & 0.0331 & 0.0294 & 0.0205 \\
& 0.6 & 0.0462 & 0.0331 & 0.0330 & 0.0396 & 0.03167 & 0.0890 \\
\hline
\end{tabular}

Table 5. Estimates of results using ADI and Crank Nicolson schemes, with reducing step size.

\begin{tabular}{cccccccc}
\hline & \multicolumn{4}{c}{$\beta_{1}=\beta_{2}=1$} & \multicolumn{3}{c}{$\beta_{1}=\beta_{2}=1 / 4$} \\
\hline \multirow{2}{*}{ Grid } & Step Size & $L_{2}$ & $L_{\infty}$ & $L_{\text {relative }}$ & $L_{2}$ & $L_{\infty}$ & $L_{\text {relative }}$ \\
\hline \multirow{2}{*}{$61 \times 61$} & $h$ & 0.0197 & 0.0117 & 0.00585 & 0.0205 & 0.01897 & 0.00889 \\
& $h / 2$ & 0.0027 & 0.0021 & 0.000675 & 0.0089 & 0.0051 & 0.000989 \\
& $h / 8$ & 0.0012 & 0.00096 & 0.00015 & 0.0021 & 0.00099 & 0.00049 \\
\hline
\end{tabular}

scheme at very small step spacing to understand the importance of reducing steps. Rate of convergence can be seen from Table 6, which explains two implicit schemes. Figure 1 \& Figure 2 show results for $\mathrm{CN}$ for different times and grids respectively. Figure 3 \& Figure 4 show results for ADI for different times and grids respectively. Figure 5 gives comparison of two implicit schemes for reducing step spacing in $x$ and $y$ directions respectively. Last Figure 6 shows interesting results for different times. Sharp edges remove during increasing 
Table 6. Presents results using ADI and CN schemes, with rate of convergence.

\begin{tabular}{cccccccc}
\hline & & \multicolumn{3}{c}{ CN Scheme } & \multicolumn{3}{c}{ ADI Scheme } \\
\hline \multirow{2}{*}{ Time $t=1$} & Grid & Rate & Rate & Rate & Rate & Rate & Rate \\
& & $L_{2}$ & $L_{\infty}$ & $L_{\text {relative }}$ & $L_{2}$ & $L_{\infty}$ & $L_{\text {relative }}$ \\
\hline \multirow{2}{*}{1} & $41 \times 41$ & 2.9385 & - & - & - & - & - \\
& $101 \times 101$ & 2.9187 & 1.6739 & 1.001 & 2.1131 & 1.0843 & 0.0471 \\
& $201 \times 201$ & 1.231 & 1.1129 & 1.0009 & 1.0938 & 1.0627 & 0.0710 \\
\hline
\end{tabular}
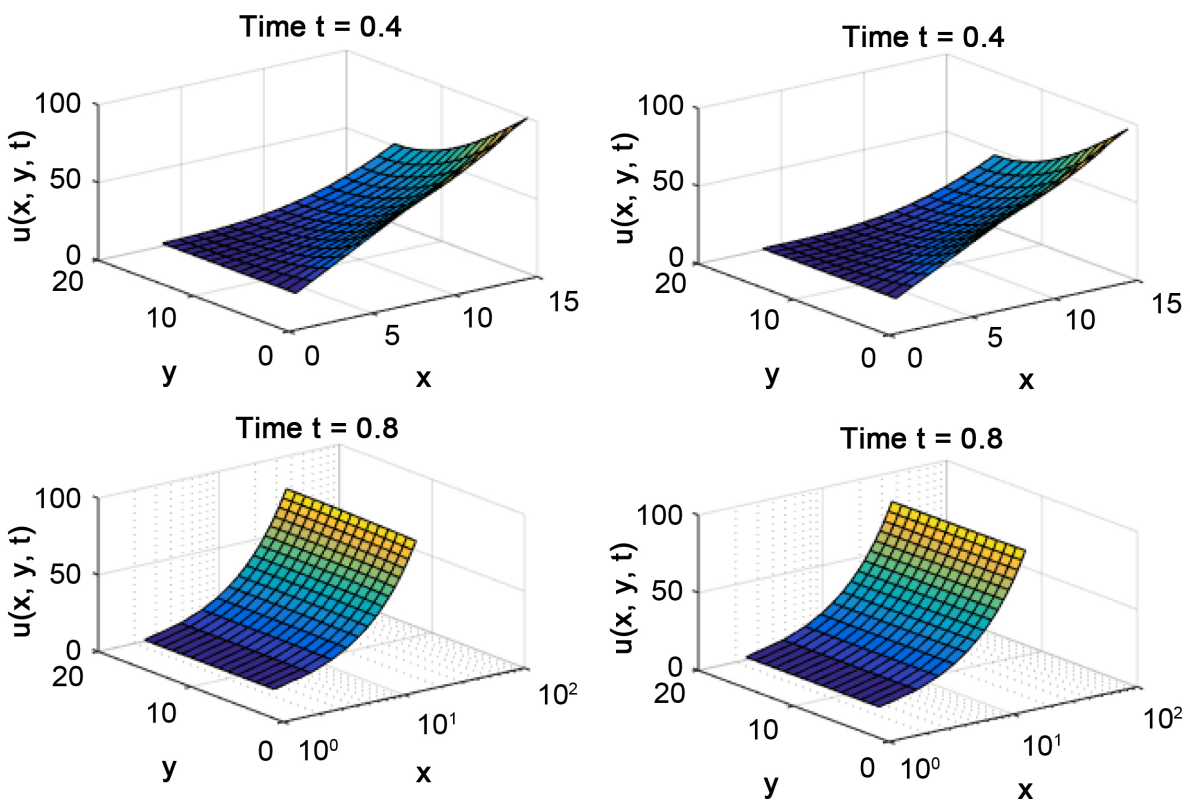

Figure 1. Shows results using $\mathrm{CN}$ scheme, at different time levels, fixed some parameters as we mentioned in Table 1.
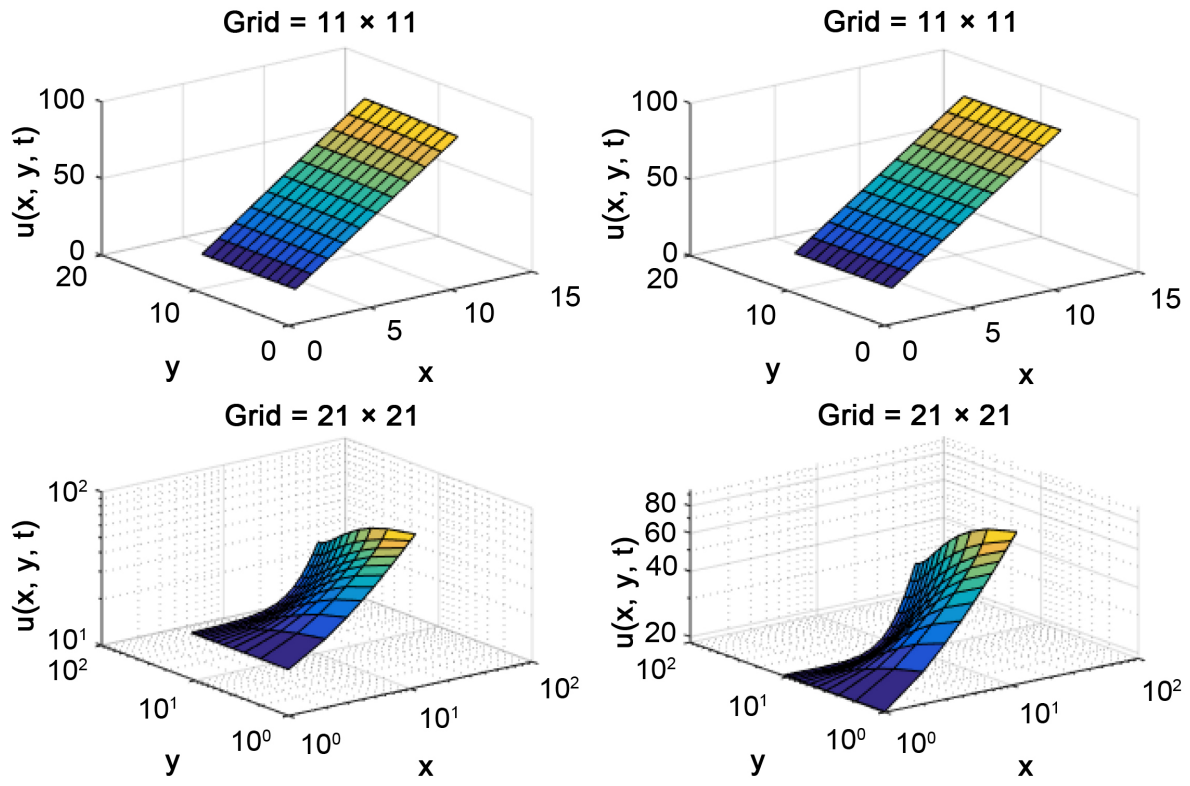

Figure 2. Shows results using $\mathrm{CN}$ scheme, at different grids, fixed some parameters as we mentioned in Table $1 \&$ Table 2. 
Grid $=91 \times 91$
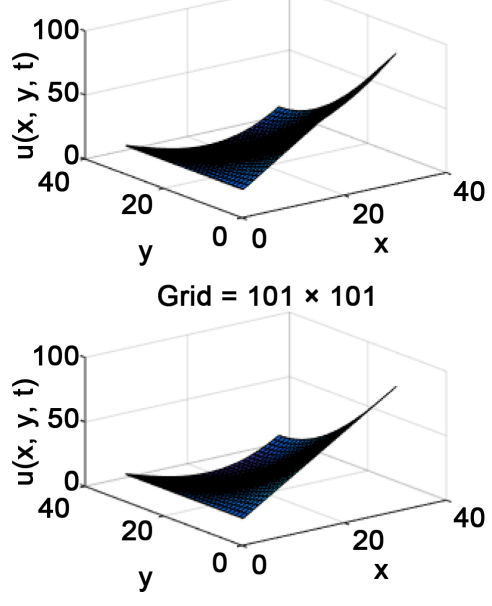

Grid $=91 \times 91$
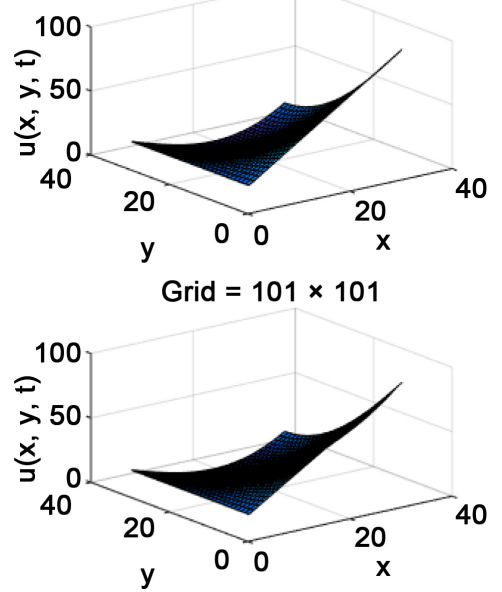

Figure 3. Shows results using ADI scheme, at different grids, fixed some parameters as we mentioned in Table 3 \& Table 4.
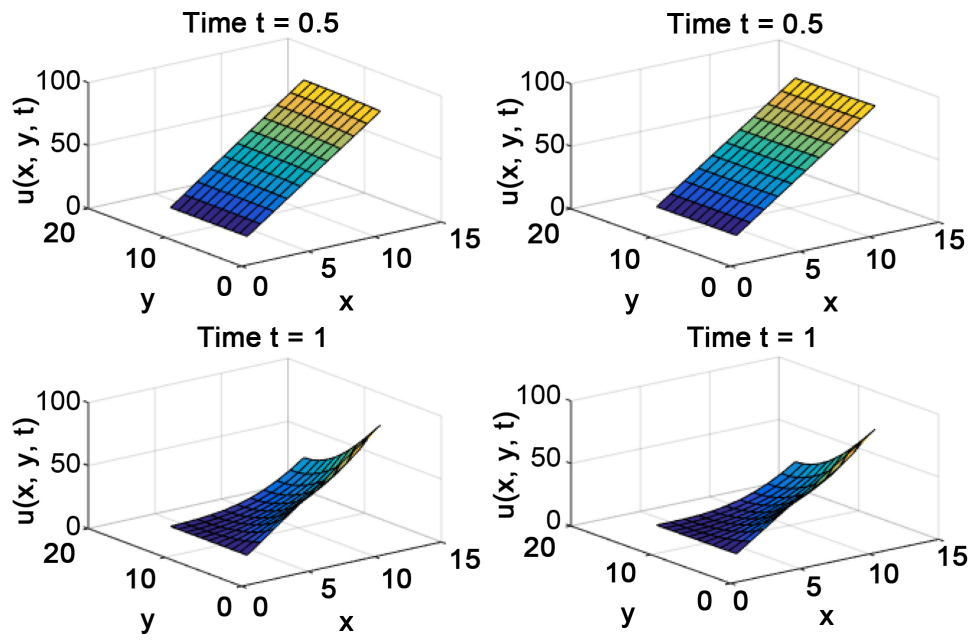

Figure 4. Shows results using ADI scheme, at different time levels, fixed some parameters as we mentioned in Table 3 \& Table 4.
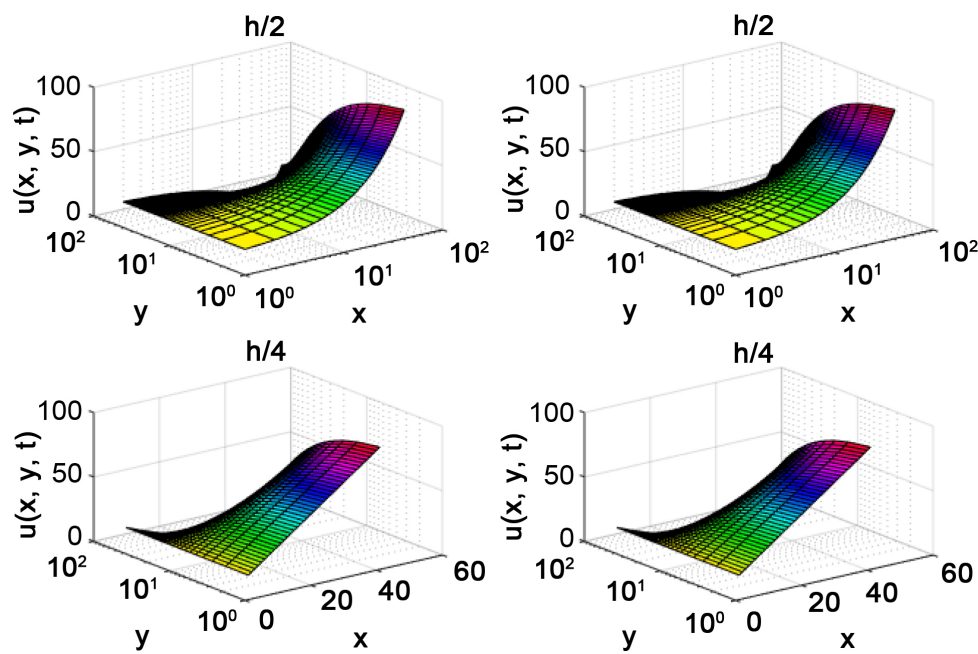

Figure 5. Shows results using ADI scheme, for two different $h$. See Table 5. 

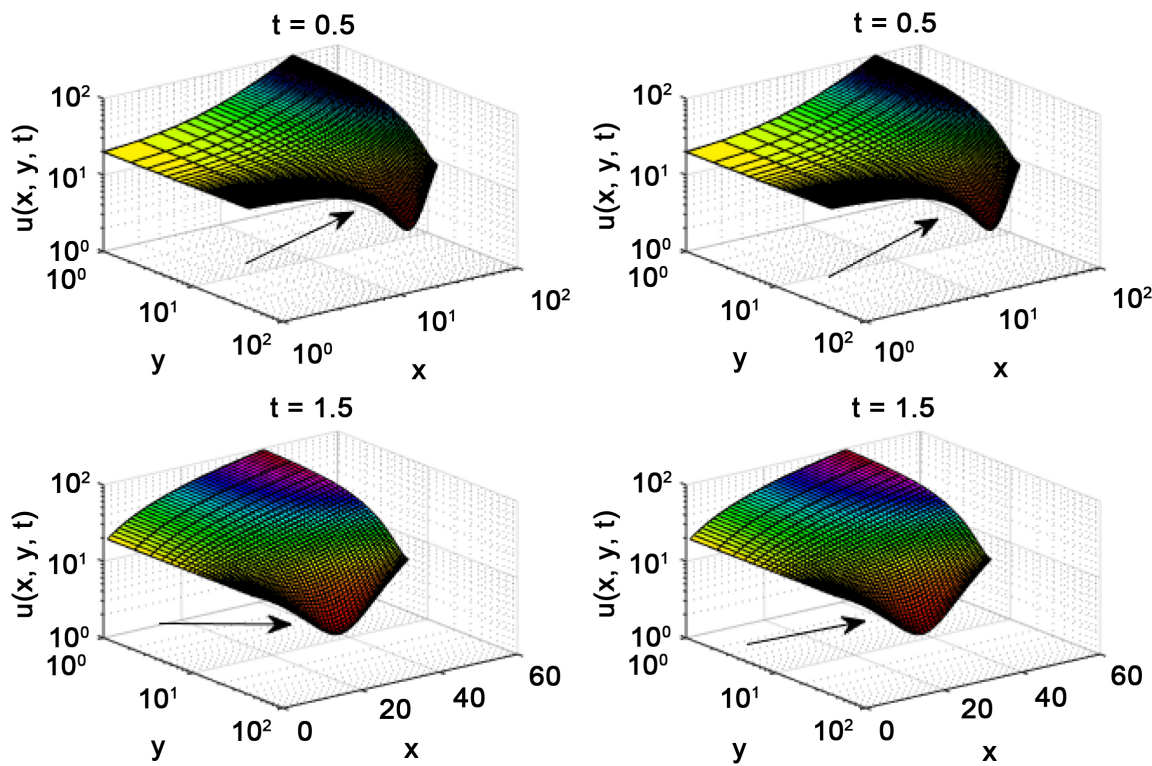

Figure 6. Shows results at different time for simple error in the concentration of the diffusion reaction phenomena. As we mentioned in this paper $u(x, y, t)$ be the concentration of the chemical. With step-up in grid size, make significant change in error but incremental in time, increase error as we can see from this figure. With increase in time, reduce the sharp edge as we mentioned in figure by arrows. These results are very interesting during simulations.

time level [31] [32] [33] [34]. These results are very interesting for us to understand the efficency of the later scheme.

\section{References}

[1] Fisher, R.A. (1936) The Wave of Advance of Advantageous Genes. Annals of Human Genetics, 7, 355-369. https://doi.org/10.1111/j.1469-1809.1937.tb02153.x

[2] Kolmogorov, A.K., Petrovsky, N.P. and Piscounov, S.P. (1937) Etude de I equations de la diffusion avec croissance de la quantitate de matiere et son application a un probolome biologique. Bull. Univ. Mosku, 1, 1-25.

[3] Newman, W.I. (1980) Some Exact Solutions to a Non-Linear Diffusion Problem in Population Genetics and Combustion. Journal of Theoretical Biology, 85, 325-334.

[4] Ronson, D.G. and Weinberger, H.F. (1975) Nonlinear Diffusion in Population Genetics, Combustion, and Nerve Pulse Propagation. Springer-Verlag., Berlin, 5-49. https://doi.org/10.1007/BFb0070595

[5] Franak Kameneetiskii, D.A. (1969) Diffusion and Heat Transfer in Chemical Kinetics. 1st Edition, Plenum Press, New York.

[6] Canosa, J.C. (1973) On a Nonlinear Diffusion Equation Describing Population Growth. IBM Journal of Research Development, 17, 307-313. https://doi.org/10.1147/rd.174.0307

[7] Arnold, R.A., Showalter, K. and Tyson, J.J. (1987) Propagation of Chemical Reactions in Space. Journal of Chemical Education, 64, 740-7744. https://doi.org/10.1021/ed064p740

[8] Tuckwell, H.C. (1988) Introduction to Theoretical Neurobiology. Cambridge University Press, Cambridge.

[9] Argyris, J.A., Haase, M.H. and Heinrich, J.C. (1991) Finite Element Approximation 
to Two-Dimensional Sine Gordon Equations. Computer Methods in Applied Mechanics and Engineering, 86, 1-26.

[10] Aronson, D.G. and Weinberger, H.F. (1978) Multidimensional Non-Linear Diffusion Arising in Population Genetics. Advance in Mathematics, 30, 33-76.

[11] Grimshaw, R.G. and Tang, S.T. (1990) The Rotation-Modified Kadomtsev-Petviashvili Equation: An Analytical and Numerical Study. Studies in Applied Mathematics, 83, 223-248. https://doi.org/10.1002/sapm1990833223

[12] Tang, S.T., Qin, S.Q. and Weber, R.O. (1991) Numerical Solution of a Non-Linear Reaction Diffusion Equation. Applied Mathematics and Mechanics, 12, 751-758.

[13] Tang, S.T. and Weber, R.O. (1991) Numerical Study of Fisher's Equation by a Petrov-Galerkin Finite Element Method. The ANZIAM Journal, 33, 27-38. https://doi.org/10.1017/S0334270000008602

[14] Williams, S.W. and Chow, P.L. (1978) Nonlinear Reaction-Diffusion Models for Interacting Populations. Journal of Mathematical Analysis and Applications, 62, 157-169.

[15] Maynard Smith, J.M. (1971) Models in Ecology. Cambridge University Press, Cambridge.

[16] Hilborn, R.H. (1975) The Effect of Spatial Heterogeneity on the Persistence of Predator-Prey Interactions. Theoretical Population Biology, 8, 346-355.

[17] Burgers, J.M. (1948) A Mathematical Model Illustrating the Theory of Turbulence. In: Advances in Applied Mechanics, Vol. 1, Academic Press, Inc., New York, 171199.

[18] Bateman, H.B. (1915) Some Recent Researches on the Motion of Fluids. Monthly Weather Review, 43, 163-170. https://doi.org/10.1175/1520-0493(1915)43<163:SRROTM>2.0.CO;2

[19] Wang, X.Y. (1988) Exact and Explicit Solitary Wave Solutions for the Generalized Fisher Equation. Physics Letters A, 131, 227-279.

[20] Gazdag, J.G. and Canosa, J.C. (1974) Numerical Solutions of Fisher's Equation. Journal of Applied Probability, 11, 445-457. https://doi.org/10.1017/S0021900200096236

[21] Logan, D.J. (1994) An Introduction to Nonlinear Partial Differential Equations. Wiley, New York.

[22] Evans, D.J. and Sahimi, M.S. (1989) The Alternating Group Explicit (AGE) Iterative Method to Solve Parabolic and Hyperbolic Partial Differential Equations. Annals of Numerical Fluid Mechanics and Heat Transfer, 2, 283-389.

[23] Ames, W.F. (1965) Nonlinear Partial Differential Equations in Engineering. Academic Press, New York.

[24] Ames, W.F. (1969) Finite Difference Methods for Partial Differential Equations. Academic Press, New York.

[25] Noye, J.N. (1981) Nonlinear Partial Differential Equations in Engineering. NorthHolland Publishing Comp. Conference in Queen's College, University of Melbourne, Australia.

[26] Ablowitz, M.J. and Zeppetella, A.Z. (1979) Explicit Solutions of Fisher's Equation for a Special Wave Speed. Bulletin of Mathematical Biology, 41, 835-840. https://doi.org/10.1007/BF02462380

[27] Wasow, W.W. (1955) Discrete Approximation to Elliptic Differential Equations. Zeitschrift für Angewandte Mathematik und Physik ZAMP, 6, 81-97.

[28] Schiesser, W.E. and Griffiths, G.W. (2009) A Compendium of Partial Differential 
Equation Models. Cambridge University Press, Cambridge. https://doi.org/10.1017/CBO9780511576270

[29] Whitham, G.B. (1974) Linear and Nonlinear Waves. John Wiley \& Sons, Hoboken.

[30] Babuska, I.B. (1968) Numerical Stability in Mathematical Analysis. IFIP Congress, North-Holland, Amsterdam, 11-23.

[31] Kanti, P.K. and Lajja, V.L. (2011) A Note on Crank-Nicolson Scheme for Burgers Equation. Applied Mathematics, 2, 888-899.

[32] Roache, P.J. (1972) Computational Fluid Dynamics. Hermosa, Albuquerque.

[33] Mazumder, S.M. (2015) Numerical Methods for Partial Differential Equations: Finite Difference and Finite Volume Methods. Academic Press, New York.

[34] Srivastava, V.K. and Tamsir, M.T. (2012) Crank Nicolson Semi-Implicit Approach for Numerical Solution of Two Dimensional Coupled Nonlinear Burgers Equations. International Journal of Applied Mechanics and Engineering, 17, 571-581.

\section{Scientific Research Publishing}

Submit or recommend next manuscript to SCIRP and we will provide best service for you:

Accepting pre-submission inquiries through Email, Facebook, LinkedIn, Twitter, etc. A wide selection of journals (inclusive of 9 subjects, more than 200 journals)

Providing 24-hour high-quality service

User-friendly online submission system

Fair and swift peer-review system

Efficient typesetting and proofreading procedure

Display of the result of downloads and visits, as well as the number of cited articles

Maximum dissemination of your research work

Submit your manuscript at: http://papersubmission.scirp.org/

Or contact ajcm@scirp.org 